\title{
Intensity-based 3D/2D registration for percutaneous intervention of major aorto-pulmonary collateral arteries
}

Julien Couet, David Rivest-Henault, Joaquim Miro, Chantal Lapierre, Luc Duong, et al.

Julien Couet, David Rivest-Henault, Joaquim Miro, Chantal Lapierre, Luc Duong, Mohamed Cheriet, "Intensity-based 3D/2D registration for percutaneous intervention of major aorto-pulmonary collateral arteries," Proc. SPIE 8316, Medical Imaging 2012: Image-Guided Procedures, Robotic Interventions, and Modeling, 831629 (17 February 2012); doi: 10.1117/12.911468 


\title{
Intensity-based $3 \mathrm{D} / 2 \mathrm{D}$ registration for percutaneous intervention of major aorto-pulmonary collateral arteries
}

\author{
Julien Couet $^{1}$, David Rivest-Hénault ${ }^{1}$, Joaquim Miró $^{2}$, Chantale Lapierre ${ }^{2}$, Luc Duong ${ }^{1}$ and \\ Mohamed Cheriet ${ }^{1}$ \\ ${ }^{1}$ École de technologie supérieure, \\ 1100 Rue Notre-Dame Ouest Montréal, H3C 1K3 Québec Canada \\ ${ }^{2}$ Centre Hospitalier Sainte-Justine, \\ 3175 Chemin de la Côte Sainte-Catherine Montréal, H3T 1C5 Québec Canada
}

\begin{abstract}
Percutaneous cardiac interventions rely mainly on the experience of the cardiologist to safely navigate inside soft tissues vessels under X-ray angiography guidance. Additional navigation guidance tool might contribute to improve reliability and safety of percutaneous procedures. This study focus on major aorta-pulmonary collateral arteries (MAPCAs) which are pediatric structures. We present a fully automatic intensity-based 3D/2D registration method that accurately maps pre-operatively acquired 3D tomographic vascular data of a newborn patient over intra-operatively acquired angiograms. The tomographic dataset 3D pose is evaluated by comparing the angiograms with simulated X-ray projections, computed from the pre-operative dataset with a proposed splatting-based projection technique. The rigid 3D pose is updated via a transformation matrix usually defined in respect of the $\mathrm{C}$-Arm acquisition system reference frame, but it can also be defined in respect of the projection plane local reference frame. The optimization of the transformation is driven by two algorithms. First the hill climbing local search and secondly a proposed variant, the dense hill climbing. The latter makes the search space denser by considering the combinations of the registration parameters instead of neighboring solutions only. Although this study focused on the registration of pediatric structures, the same procedure could be applied for any cardiovascular structures involving CT-scan and X-ray angiography. Our preliminary results are promising that an accurate (3D TRE $0.265 \pm 0.647 \mathrm{~mm})$ and robust $(99 \%$ success rate) bi-planes registration of the aorta and MAPCAs from a initial displacement up to $20 \mathrm{~mm}$ and $20^{\circ}$ can be obtained within a reasonable amount of time (13.7 seconds).
\end{abstract}

Keywords: 3D/2D Registration, Image-Guided Interventions, Cardiac Procedures

\section{INTRODUCTION}

Multimodal 3D/2D registration has multiple applications in assisting medical diagnosis and therapy, from data fusion from different acquisition modalities to radiographic-based navigation guidance. The aim of 3D/2D registration is to define a common reference frame for multimodal medical datasets in order to align corresponding anatomical structures of the patient. The motivation of this work is to improve minimally invasive treatment of major aorta-pulmonary collateral arteries (MAPCAs) in pediatric cardiology. MAPCAs are immature arteries with blob morphology associated with severe congenital heart diseases as the pulmonary atresia with ventricular septal defect or the Tetralogy of Fallot, observed in newborn patients. ${ }^{1}$ Furthermore, MAPCAs are very thin vascular structures $^{2}$ (their diameter is smaller than $4 \mathrm{~mm}$ ) that can be highly complex in 3D and thus extremely challenging to navigate. By mapping a pre-operative segmented 3D tomographic dataset of MAPCAs onto X-ray intra-operative angiographies, we aimed at improving X-ray angiography-based navigation of MAPCAs for pediatric patients. The first purpose of this work is to investigate whether rigid registration of small was possible, and the second is to compensate rigid respiratory movements of such vascular structures. This study is focused on pediatric cardiology, but could be adapted to any adult percutaneous procedure in cardiology involving tomographic data and X-ray angiographies such as aortic or coronary diseases.

Further author information:

Julien Couet : E-mail: julien.couet.1@ens.etsmtl.ca.

\footnotetext{
Medical Imaging 2012: Image-Guided Procedures, Robotic Interventions, and Modeling, edited by David R. Holmes III, Kenneth H. Wong, Proc. of SPIE Vol. 8316, 831629 (c) 2012 SPIE $\cdot$ CCC code: $1605-7422 / 12 / \$ 18 \cdot$ doi: $10.1117 / 12.911468$
} 
Intensity-based rigid $3 \mathrm{D} / 2 \mathrm{D}$ registration methods described in the literature are mainly developed for adult decease interventions. ${ }^{3}$ The common workflow relies on the simulation of X-ray angiographies from the preoperative tomographic data at an estimated 3D pose. This process is known as digitally reconstructed radiographs (DRR) that is mainly achieved by voxels projection ${ }^{4}$ or ray tracing techniques. ${ }^{5}$ DRRs are iteratively compared with the reals angiograms via an intensity-based similarity measure. ${ }^{6}$ The $3 \mathrm{D}$ rigid pose of the CT data is then updated until the similarity measure is optimal. This non-linear 6 degrees of freedom problem is commonly solved by the use of local iterative optimization algorithms as the hill climbing, ${ }^{7}$ Powell-based $^{8}$ and the downhill simplex ${ }^{9}$ algorithms or first and second order derivative-based optimization as the Levenberg-Marquardt ${ }^{10}$ and the L-BFGS. ${ }^{11}$ To converge, these algorithms theoretically require monotone and convex objective cost functions in the vicinity of the true position. However, none of the current similarity measures respects theses properties which may result in the optimization process to diverge towards a local maxima. Hence, intensity-based methods have a small capturing range and must be initialized close to the true pose. Markelj et al. ${ }^{3}$ identifies two mains approaches, regardless the optimization algorithm, to increase the searched space while avoiding false local optima. The first involves the use of multi-resolution and/or multi-scale search strategies. However downsampling or smoothing the images may, at the same time, increase risks of trapping the optimization within a false optimum. The second intends to bring the registration problem within the capture range of the optimization algorithm by sequential/parallel multi-initialization strategies or by iteratively decreasing a wide initial searched space. Nevertheless, these methods appear being computationally expensive.

In this paper, we present a novel biplane automatic intensity-based $3 \mathrm{D} / 2 \mathrm{D}$ rigid registration workflow that accurately maps pre-operative CT data with intra-operative X-ray angiographies in order to assist percutaneous intervention of pediatric congenital heart diseases. First, an improvement over the wobbled voxels splatting method $^{12}$ is proposed. Secondly, to improve the accuracy of the registration, the rigid transformation that maps the $3 \mathrm{D}$ pre-operative and the $2 \mathrm{D}$ intra-operative datasets is expressed within the coordinate frame of one of the C-Arm projection plans (the intensifiers) instead of within the coordinate frame of the radiographic acquisition system. Finally, to increase the robustness of the registration technique, a new local iterative optimization algorithm, based on the hill climbing, ${ }^{7}$ is proposed.

\section{METHODS}

\subsection{DRRs simulation}

DRRs are computed by projecting the volumetric CT dataset onto two different simulated projection planes, the registration being biplane to be more accurate. To be computationally efficient, the X-ray images are simulated with a voxels splatting method instead of a ray tracing technique. ${ }^{12}$ The main drawback of splattingbased projection is the presence of aliasing artifacts forming undesirable lines. ${ }^{4}$ To increase the DRRs quality, Birkfellner et al. ${ }^{12}$ used an anti-aliasing technique composed of a random wobbled vibration of the voxels and/or the pixels positions coupled with a gaussian smoothing. Nevertheless, discretization artifacts remain persistent with the the tomographic dataset used in this work, as shown in Figure 1(a). Furthermore, the wobbled vibration introduces a noise that the gaussian kernel cannot properly smooth with our volumetric projected data.

In order to define a splatting-based projection method usable for our registration framework, i.e to decrease the amount of artifacts to obtain high quality DRRs, two solutions are proposed. The first is to replace the gaussian filter with the convolution of triangles kernel ${ }^{13}$ (COT) which appears to strongly erase both the artifacts and the noise introduced by the vibrations, as shown in Figure 1(b). The second is to compute the length of each projection ray within the voxels it reaches as in a ray tracing technique. ${ }^{5}$ However, the computation of the exact path of a ray within a tomographic dataset is computationally expensive. Instead, we choose to compute an average $l_{i}$ length for all the voxels that a ray $\overrightarrow{\mathbf{p}_{\mathbf{i}}} \overrightarrow{\mathbf{s}}$ crossed through. As shown in Figure $1(\mathrm{c})$, a mean ray length $d$ is first computed from a voxel along of the optical axe $\overrightarrow{\mathbf{p}_{\mathbf{o}} \mathbf{s}}$ and then projected on the current projection ray $\overrightarrow{\mathbf{p}_{\mathrm{i}} \mathbf{s}}$, cf. equation (1).

$$
l_{i}=d \cdot \cos \left(\alpha_{i}\right) \quad \cos \left(\alpha_{i}\right)=\frac{\overrightarrow{\mathbf{p}_{\mathbf{o}} \mathbf{s}} \cdot \overrightarrow{\mathbf{p}_{\mathbf{i}} \mathbf{S}}}{\left\|\overrightarrow{\mathbf{p}_{\mathbf{o}} \mathbf{s}}\right\| \cdot \| \overrightarrow{\mathbf{p}_{\mathbf{i}} \mathbf{S} \|}}
$$

As illustrated in Figure $1(\mathrm{c})$, whereas the lengths $\left(l_{i_{1}} . . l_{i_{4}}\right)$ are different in a ray tracing technique, these lengths are all equal in the proposed splatting-based DRR computation method. 


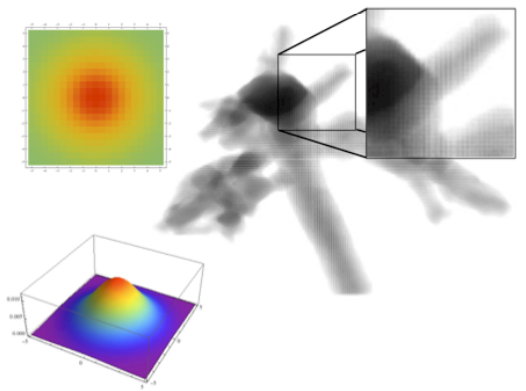

(a) Gaussian kernel.

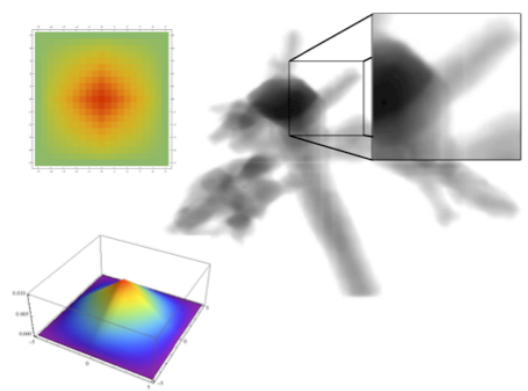

(b) COT kernel.

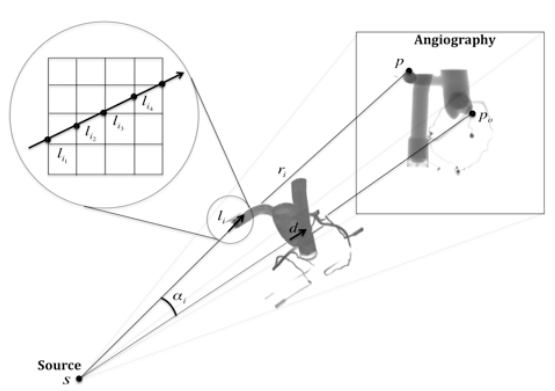

(c) Projection ray length computation.

Figure 1: Improved wobbled-splatting DRRs computation method with the first MAPCAs tomographic dataset. (a) DRR smoothed with a 5x5 Gaussian kernel with the artifacts. (b) DRR smoothed with a 5x5 COT kernel.

(c) Computation of the projection ray length within the tomographic voxels.

\subsection{Similarity measure}

Since the registration is quasi-multimodal, ${ }^{3}$ the similarity between the target $I_{t}$ and the registered radiographs $I_{r}$ is quantified with the entropy correlation coefficient ${ }^{14}$ (ECC). It is theoretically maximized and equals unit when the two images share the same information. Compared to the normalized mutual information ${ }^{15}$ (NMI), the presence of the square root int the ECC definition tends to increase the amplitude of all the local optima, and particularly the true one, as shown in Figure 2(a). The final measure $\mathcal{O}$ used to minimize the optimization cost of both views $\left(I_{1}, I_{2}\right)$ is defined by equation (2), where $\left(E\left(I_{t}\right), E\left(I_{r}\right)\right)$ are the entropy and $E\left(I_{t}, I_{r}\right)$ the joint entropy of the X-ray images.

$$
\operatorname{ECC}\left(I_{t}, I_{r}\right)=\sqrt{2 \cdot\left(1-\frac{E\left(I_{t}, I_{r}\right)}{E\left(I_{t}\right)+E\left(I_{r}\right)}\right)} \quad \mathcal{O}=1-\frac{E C C\left(I_{t_{1}}, I_{r_{1}}\right)+E C C\left(I_{t_{2}}, I_{r_{2}}\right)}{2}
$$

\subsection{Expression of the rigid transformation}

The coordinate systems used in the biplane registration framework are illustrated in Figure 2(b). $\mathcal{R}_{a}$ is the fixed and absolute global reference frame defined at the C-Arm iso-center. $\mathcal{R}_{v}$ is the local reference frame of the volumetric tomographic dataset defined at its center. $\mathcal{R}_{p}$ defines the local reference frame of the first projection plane, in red in the figure. To obtain the optimal rigid pose of the tomographic data, a transformation $T$ is applied to the volumetric tomographic dataset. The transformation $T$ is represented by a $4 \mathrm{x} 4$ matrix (in homogeneous coordinates) defined from a 6 parameters vector $\mathbf{P}$ representing the $(\vec{x}, \vec{y}, \vec{z})$ axis translations and rotations components : $\mathbf{P}=\left(t_{x}, t_{y}, t_{z} ; \theta_{x}, \theta_{y}, \theta_{z}\right)^{\top}$. Rotations are uniquely factorized in Euler form since their amplitudes are always inferior to $90^{\circ 7}$ and performed at the center of the tomographic dataset. The optimization of the rotation parameters in the local reference frame is preferred over the global reference frame, since it ensures that neighboring transformations will be spatially coherent in 3D space.

In the conventional scenario, the transformation $T$ is defined with the respect of $\mathcal{R}_{a}$. However, $T$ can also be defined with the respect of the local reference frame of one of the projection plane. We choose the first projection plane where the reference frame is $\mathcal{R}_{p}$ and the transformation now represented by $T^{p_{\text {ref }}}$. The second projection plane is conventionally used to further constraint the volume 3D pose from an different view. To apply $T^{p_{r e f}}$ to the local reference frame of the CT volume $\mathcal{R}_{v}$, the rigid transformation has to be first converted in respect of $\mathcal{R}_{a}$. The new transformation $T^{a_{r e f}}$ is then defined by equation (3) where $T_{p}$ is the transformation matrix of $\mathcal{R}_{p}$ in respect of $\mathcal{R}_{a}, T_{p}^{-1}$ its inverse.

$$
T^{a_{r e f}}=T_{p}^{-1} \cdot T^{p_{r e f}} \cdot T_{p}
$$

This definition allows to move and rotate the volumetric $\mathrm{CT}$ data in the directions of $\mathcal{R}_{p}$ axes instead of the ones of $\mathcal{R}_{a}$. By defining the transformations as a mapping from $\mathcal{R}_{v}$ to $\mathcal{R}_{p}$, the influence on the similarity measure of each single parameter transformation is better discriminated and the registration precision improved. 


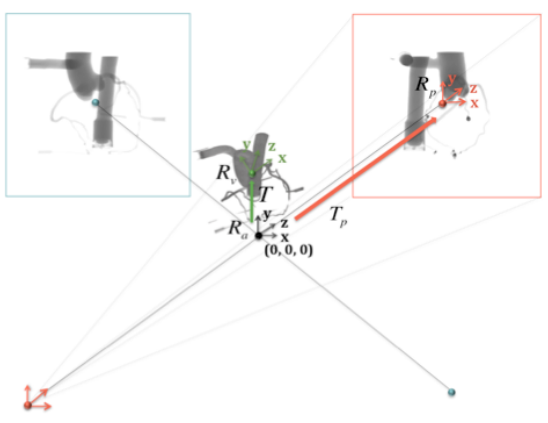

(a) Registration geometry.

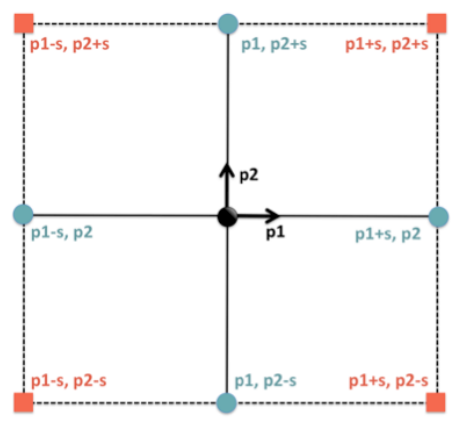

(b) Optimization scheme.

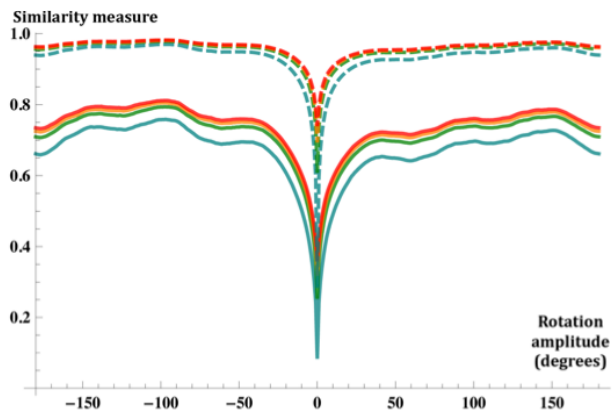

(c) ECC and NMI noise robustness.

Figure 2: Registration workflow schemes : (a) Geometry of the biplane imaging system representing an anteroposterior view and a lateral view ${ }^{16}$ (the two planes are perpendicular) employed in the registration framework. (b) 2D HC and DHC search schemes comparison. Positions reached by the $\mathrm{HC}$ are the blue points while the positions attained by the DHC are both the blue points and the red squares. (c) Robustness of the NMI and the ECC similarity measures to a gaussian white noise with the second dataset of MAPCAs. The CT data is rotated from $\theta_{x}=-180^{\circ}$ to $\theta_{x}=180^{\circ}$ along the $\vec{x}$ axe of the reference frame $\mathcal{R}_{a}$, a DRR is created at every degree and then compared to the image of reference computed at $\theta_{x}=0$. The dash lines represent the results for the NMI similarity measure while the plan lines depict the ECC measure. The colors represents the intensity of the white gaussian noise, from blue where there is no noise to red were the angiographies are very noisy.

\subsection{Optimization strategy}

In the hill climbing (HC) algorithm, ${ }^{7}$ the local search is carried out by sequentially altering, at every iteration $t$, the position of each search parameter, now represented by the general notation $\mathbf{P}=\left\{p_{i}, i \in[1,6]\right\}$, a fixed step size $s$ in both negative and positive directions as shown in equation (4).

$$
\left(p_{i}^{t}-s, p_{i}^{t}+s\right) \quad i \in[1 . .6]
$$

After having evaluated all the $2 \times 6=12$ neighbors, the position improving the similarity measure the most is kept and then set as the initial position for the next iteration. To cover a larger search amplitude, a initial wide search parameters step size $s=s_{\max }$ is decreased as the iteration number increases until $s$ reachs $s_{\min }$, the stopping condition that also represents the accuracy of the registration method to achieve. The search parameters $p_{i}$ are ordered in translation-rotation components : $\mathbf{P}=\left(t_{x}, t_{y}, t_{z} ; \theta_{x}, \theta_{y}, \theta_{z}\right)^{\top}$ and always set to null at the initialization. The rigid transformation $T$ is defined in respect of the reference frame $\mathcal{R}_{a}{ }^{7}$ or in respect of $\mathcal{R}_{p}$, as presented in paragraph 2.3 .

Nevertheless, the number of the evaluations can be increased by considering all the combinations of the 6 transformation parameters instead of limiting the search to the closest neighboring locations only. As each parameter $p_{i}$ of $\mathbf{P}$ can independently take 3 positions as shown by equation (5), the combination of all the parameters, without counting the current pose, equals to $3^{6}-1=728$ (cf. figure 2.b).

$$
\left(p_{i}^{t}-s, p_{i}^{t}, p_{i}^{t}+s\right) \quad i \in[1 . .6]
$$

Although evaluating all the possibilities will be computationally very intensive, make denser the search space allows to make the volumetric dataset visit more locations and thus, increases the probability of finding the true pose. However, this approach might be affected by the intensity measure properties. Indeed, if the intensity measure is not smooth enough, visiting more locations also increases risks of trapping the optimization within a false optimum. To confirm this hypothesis while decreasing the number of locations to evaluate, we propose a variant of the hill climbing algorithm, the dense hill climbing (DHC). The local search is speeded up by dividing the search 6 parameters $p_{i}$ of $\mathbf{P}$ into two groups : translation $\left(t_{x}, t_{y}, t_{z}\right)^{\top}$ and rotation $\left(\theta_{x}, \theta_{y}, \theta_{z}\right)^{\top}$ components. At each iteration $t$, the parameters of the rotation group are first fixed and only the $3^{3}-1=26$ translations are evaluated. The translation improving the similarity measure the most is kept to next find the best rotation combination. With this search scheme, the number of evaluations to perform at each iteration then leads up to 52 evaluations compared to the initial 728 . 


\section{EXPERIMENTAL RESULTS}

\subsection{Data sets and evaluation}

Validation of the proposed registration technique was performed on three different tomographic datasets. First, an adult size and high quality coronary arteries phantom, ${ }^{17}$ with the aorta, filled with contrast agent and acquired by CT-scan (Figure 3). This dataset, composed of $512 \times 412 \times 388$ voxels, represents an ideal tomographic acquisition where the arteries are motionless and the contrast agent present within all the structure without any diffusion effects. The later are real MAPCAs CT datasets of two pediatric patients composed of $512 \times 512 \times 214$ voxels as shown in Figures 4 and 5. Theses datasets were segmented using a semi-automatic level set method. ${ }^{18}$ X-ray angiograms were simulated using $2 \mathrm{D}$ projection of the CT datasets as described above. The $3 \mathrm{D} / 2 \mathrm{D}$ targetto-registration error ${ }^{14}$ (TRE) mean and standard deviation, in millimeters, are computed with a set of $5003 \mathrm{D}$ points placed along relevant structures (aorta, vessels or MAPCAs) to create centerlines of the target vessels as shown in Figures 3 and 4. As shown in Figure 7, the segmented second dataset does not present enough MAPCAs to create a significant number of points usable for the TRE calculations. Instead, the centerlines defined for the first real pediatric dataset were used.

To validate our method, two types of experimentations were performed. First, we compared, in term of precision and bias, the proposed optimization algorithm (DHC) with the hill climbing (HC) as well as the two possible reference frames, $\mathcal{R}_{a}$ and $\mathcal{R}_{p}$, usable for defining the rigid transformation (cf. section 2.3). The target, or ground truth, poses were uniformly distributed within an maximum search amplitude $s_{\max }$ ranged from $\{ \pm 10, \pm 20, \pm 30\}$ millimeters for the translations and degrees for the rotations. For all the tests, the initial pose, from where the CT datasets had to reach the target poses, was set to null and the stopping condition $s_{m i n}$ was set to $10^{-4}$. Every test batch was composed of registrations from 100 random poses. We did not consider outlier registrations in the final 3D/2D TRE mean and standard deviation errors, a registration $i$ being defined as an outlier if its own TRE3D $(i) \leq s_{\max } / 2$. Secondly, the proposed registration technique was used to compensate a simulated one-dimensional respiratory motion ${ }^{19} r_{\vec{y}}$ along the craniocaudal direction, i.e in the direction of the $\vec{y}$ axe of the reference frame $\mathcal{R}_{a}$.

$$
r_{\vec{y}}(t)=A \cdot \cos ^{2 n}\left(\frac{\pi \cdot t}{\tau}+\phi\right)
$$

In equation (6), the parameter $A=20 \mathrm{~mm}$ is the maximum amplitude of the respiratory movement, $\tau=11 \mathrm{~s}$ and $\phi=0$ are respectively the period and the initial phase of the breathing cycle. The last coefficient $n=1$, specific to each patient, determines the breathing pattern. Two types of motions were simulated, a perfect sinusoidal movement and an altered one which is closer to a real breathing motion.

\subsection{Rigid registration results}

The Figures 3, 4 and 5 present the rigid registration results where the parameters $(\mathrm{A}, \mathrm{B}, \mathrm{C})$ represent: $(\mathrm{A})$ the maximum simulated amplitude $s_{\max }\left(\mathrm{ex} . s_{\max }=10: 10 \mathrm{~mm}\right.$ and 10 degrees), (B) the reference frame considered, (C) the optimization strategy. The parameters ( $\mathrm{t}$, No, Nc) depict: (t) the computation time in seconds without projection time and rounded up, (No): the number of outliers, and (Ni) the number of iterations of the optimization algorithms.

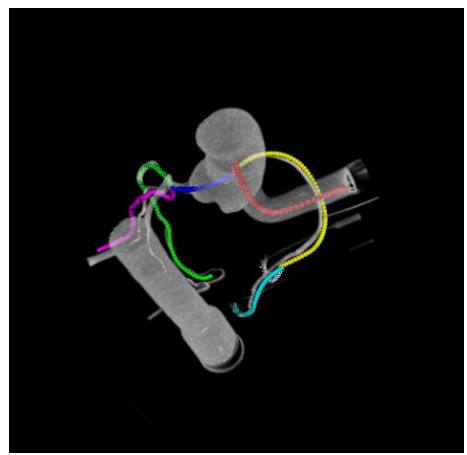

\begin{tabular}{|ccl||ccccc|}
\hline & & & \multicolumn{5}{c|}{ Phantom } \\
\hline $\mathrm{A}$ & $\mathrm{B}$ & $\mathrm{C}$ & TRE2D $(\mathrm{mm})$ & TRE3D $(\mathrm{mm})$ & $\mathrm{t}(\mathrm{s})$ & No & Nc \\
\hline \hline \hline 10 & $\mathcal{R}_{a}$ & HC & $0.089 \pm 0.634$ & $0.077 \pm 0.556$ & 13 & 0 & 671 \\
& & DHC & $0.024 \pm 0.021$ & $0.019 \pm 0.016$ & 21 & 0 & 1224 \\
& $\mathcal{R}_{p}$ & HC & $0.024 \pm 0.009$ & $0.019 \pm 0.008$ & 12 & 0 & 686 \\
& & DHC & $0.025 \pm 0.025$ & $0.082 \pm 0.085$ & 22 & 0 & 1233 \\
\hline \hline 20 & $\mathcal{R}_{a}$ & HC & $0.087 \pm 0.269$ & $0.107 \pm 0.348$ & 23 & 1 & 897 \\
& & DHC & $0.037 \pm 0.016$ & $0.027 \pm 0.015$ & 38 & 0 & 1160 \\
& $\mathcal{R}_{p}$ & HC & $0.293 \pm 1.348$ & $0.243 \pm 1.093$ & 27 & 1 & 931 \\
& & DHC & $0.032 \pm 0.021$ & $0.029 \pm 0.020$ & 42 & 1 & 1663 \\
\hline \hline 30 & $\mathcal{R}_{a}$ & HC & $0.516 \pm 1.851$ & $0.595 \pm 2.146$ & 32 & 11 & 1269 \\
& & DHC & $0.275 \pm 1.345$ & $0.302 \pm 1.562$ & 45 & 4 & 2343 \\
& $\mathcal{R}_{p}$ & HC & $0.204 \pm 0.850$ & $0.462 \pm 2.119$ & 26 & 9 & 1197 \\
& & DHC & $0.148 \pm 0.517$ & $0.161 \pm 0.645$ & 44 & 1 & 2315 \\
\hline
\end{tabular}

Figure 3: Evaluation of the precision of the 3D/2D registration of the adult-size synthetic aortic phantom. 


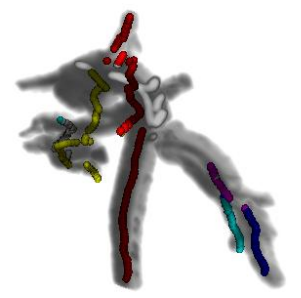

\begin{tabular}{|c|c|c|c|c|c|c|c|}
\hline & & & & MAPCAs-1 & & & \\
\hline $\mathrm{A}$ & $\mathrm{B}$ & $\mathrm{C}$ & TRE2D $(\mathrm{mm})$ & TRE3D $(\mathrm{mm})$ & $\mathrm{t}(\mathrm{s})$ & No & $\mathrm{Nc}$ \\
\hline 10 & $\mathcal{R}_{A}$ & $\mathrm{HC}$ & $0.062 \pm 0.039$ & $0.054 \pm 0.034$ & 12 & 0 & 735 \\
\hline & & $\mathrm{DHC}$ & $0.568 \pm 0.982$ & $0.487 \pm 0.853$ & 20 & 0 & 1298 \\
\hline & $\mathcal{R}_{P}$ & $\mathrm{HC}$ & $0.062 \pm 0.054$ & $0.053 \pm 0.045$ & 13 & 0 & 723 \\
\hline & & $\mathrm{DHC}$ & $0.066 \pm 1.178$ & $0.581 \pm 1.065$ & 13 & 0 & 1268 \\
\hline 20 & $\mathcal{R}_{A}$ & $\mathrm{HC}$ & $0.492 \pm 1.204$ & $0.435 \pm 1.115$ & 18 & 6 & 1460 \\
\hline & & $\mathrm{DHC}$ & $2.891 \pm 3.276$ & $2.473 \pm 2.752$ & 20 & 12 & 1593 \\
\hline & $\mathcal{R}_{P}$ & $\mathrm{HC}$ & $0.265 \pm 0.647$ & $0.236 \pm 0.607$ & 14 & 1 & 1050 \\
\hline & & $\mathrm{DHC}$ & $2.305 \pm 2.787$ & $1.954 \pm 2.394$ & 27 & 8 & 1612 \\
\hline 30 & $\overline{\mathcal{R}_{A}}$ & $\mathrm{HC}$ & $0.961 \pm 2.581$ & $0.850 \pm 2.240$ & 18 & 46 & 1441 \\
\hline & & $\mathrm{DHC}$ & $3.360 \pm 4.122$ & $2.942 \pm 3.648$ & 14 & 32 & 2440 \\
\hline & $\mathcal{R}_{P}$ & $\mathrm{HC}$ & $0.995 \pm 2.149$ & $0.869 \pm 1.867$ & 21 & 41 & 1443 \\
\hline & & $\mathrm{DHC}$ & $5.529 \pm 4.812$ & $4.703 \pm 4.095$ & 25 & 21 & 2178 \\
\hline
\end{tabular}

Figure 4: Evaluation of the precision of the $3 \mathrm{D} / 2 \mathrm{D}$ registration of the first real pediatric dataset of MAPCAs.

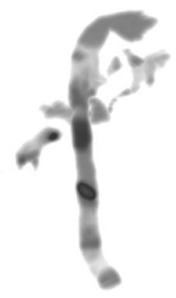

\begin{tabular}{|cll||ccccc|}
\hline \multicolumn{1}{|c||}{} & \multicolumn{5}{c|}{ MAPCAs-2 } \\
\hline $\mathrm{A}$ & $\mathrm{B}$ & $\mathrm{C}$ & TRE2D $(\mathrm{mm})$ & TRE3D $(\mathrm{mm})$ & $\mathrm{t}(\mathrm{s})$ & No & Nc \\
\hline \hline \hline 10 & $\mathcal{R}_{A}$ & HC & $1.121 \pm 1.596$ & $0.962 \pm 1.375$ & 12 & 0 & 815 \\
& & DHC & $2.026 \pm 2.388$ & $1.735 \pm 2.038$ & 16 & 1 & 1255 \\
& $\mathcal{R}_{P}$ & HC & $0.839 \pm 1.145$ & $0.715 \pm 0.977$ & 14 & 1 & 879 \\
& & DHC & $2.265 \pm 1.178$ & $1.895 \pm 2.042$ & 14 & 3 & 1260 \\
\hline \hline \hline 20 & $\mathcal{R}_{A}$ & HC & $2.385 \pm 3.123$ & $2.060 \pm 2.712$ & 25 & 17 & 1075 \\
& & DHC & $4.548 \pm 3.243$ & $3.709 \pm 2.648$ & 38 & 27 & 1540 \\
& $\mathcal{R}_{P}$ & HC & $1.595 \pm 2.131$ & $1.377 \pm 1.837$ & 16 & 18 & 1135 \\
& & DHC & $4.248 \pm 3.093$ & $3.494 \pm 2.566$ & 29 & 28 & 1479 \\
\hline
\end{tabular}

Figure 5: Evaluation of the precision of the $3 \mathrm{D} / 2 \mathrm{D}$ registration of the second dataset of MAPCAs. The results for $s_{\max }= \pm 30$ are not presented since the number of outlier reach $50 \%$ of the tests, and thus are not significant.

While the most precise results, in term of 3D TRE but mainly in term of number of outliers, are obtained with the dense hill climbing (DHC) algorithm with the synthetic CT dataset $(0.161 \pm 0.645 \mathrm{~mm}$ with $1 \%$ of outliers and $\left.s_{\max }= \pm 30\right)$, the hill climbing algorithm ${ }^{7}(\mathrm{HC})$ provides better results when dealing with the real pediatric CT datasets $(0.236 \pm 0.607 \mathrm{~mm}$ with with $1 \%$ of outliers for the first and $1.377 \pm 1.837 \mathrm{~mm}$ with $16 \%$ of outliers for the second with $s_{\max }= \pm 20$ ). First, that confirms that the precision of the registration depends on the quality of the projected CT dataset, which also defined the behavior of the similarity measure. Secondly, that also reinforces our assumption that taking into account more locations for the 3D CT data in the search scheme, as presented in section 2.4, is relevant only if the similarity measure presents a few number of local false optima, i.e is well behaved in the vicinity of the true pose of the CT data. Otherwise, the risks that trapping the optimization algorithm into a false local minimum increased enough to clearly limited the robustness of the registration. However, the proposed optimization algorithm, with the aortic phantom, allows the registration to be very precise with $s_{\max }=30$ and exact if $s_{\max } \leq 20$. If coupled with an appropriate similarity measure, the dense hill climbing algorithm promises to be more efficient than the hill climbing optimization.

Concerning the reference frame $\mathcal{R}_{a}$ or $\mathcal{R}_{p}$ from where the rigid transformation matrix $T$ is defined, it appears that, when associated with the proper optimization algorithm, defining the transformation in respect of the projection plane reference $\mathcal{R}_{p}$ increases significantly the accuracy of the registration by decreasing the final standard deviation of the target-to-registration error.

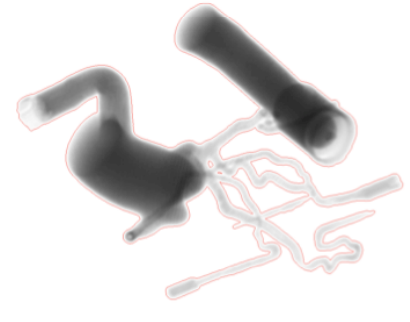

(a) Synthetic phantom

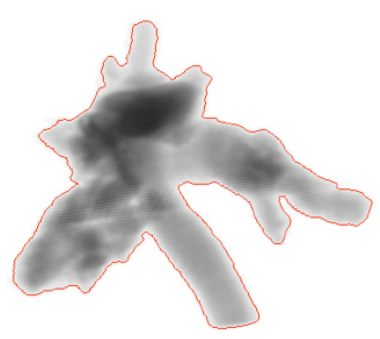

(b) First MAPCAs

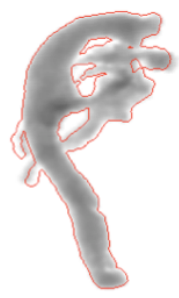

(c) Second MAPCAs

Figure 6: Examples of registration results in 2D with all the tomographic datasets. 


\subsection{Motion compensation results}

The results of the respiratory motion compensation are illustrated by the Figures $7(\mathrm{a})$ and $7(\mathrm{~b})$. The simulated respiratory motion is represented by the green points while the compensated movement is shown by the orange squares that represent the TRE3D error. The frequency of the X-ray acquisition is $0.6 \mathrm{~Hz}$ which leads to one angiography acquisition each $1.67 \mathrm{~s}$.

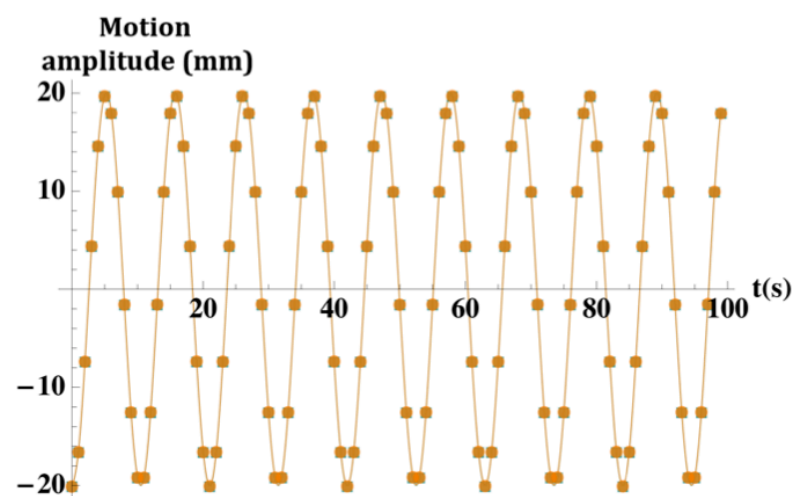

(a) Compensation of a perfect sinusoidal movement.

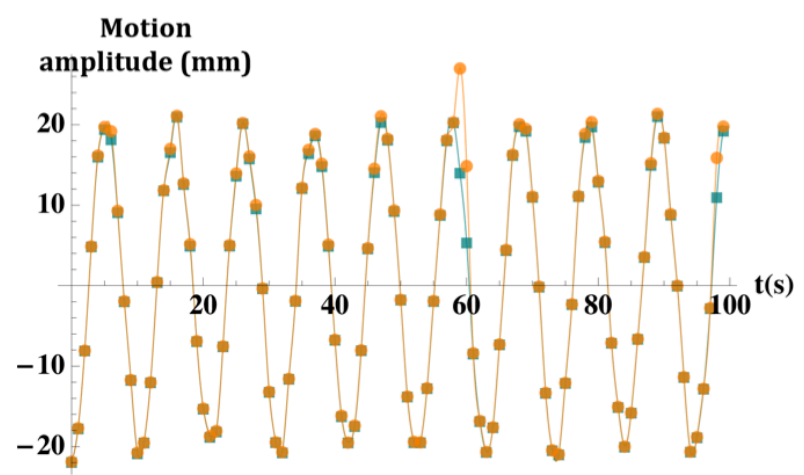

(b) Compensation of an altered movement.

Figure 7: Automatic compensation of the respiratory motion with the second dataset of MAPCAs. The compensation was performed with the hill climbing algorithm and the rigid transformations defined within the reference frame $\mathcal{R}_{p}$ of the projection plane.

The propose registration framework is able to compensate a rigid breathing motion of an amplitude of $\pm 20 \mathrm{~mm}$. While the compensation is perfect with the exactly sinusoidal motion, some errors appear with the altered motion. However, these errors are rapidly corrected and, furthermore, are not propagated.

\section{CONCLUSION}

This study aimed at presenting a 3D/2D registration workflow for pediatric cardiac interventions. Navigation using X-ray angiography during percutaneous interventions is highly challenging, since it involves mentally registering two $2 \mathrm{D}$ views of a moving 3D anatomical structure into the patient reference. This paper presents a novel technique for biplane intensity-based registration of the aortic root with MAPCAs of a pre-operative tomographic acquisition and X-ray angiography imaging. The main contribution of this work is a new $3 \mathrm{D} / 2 \mathrm{D}$ rigid registration framework that is based on three steps. First, the wobbled splatting DRR computation method ${ }^{12}$ is improved by replacing the final gaussian smoothing with the COT kernel ${ }^{13}$ and by approximating the length of the projection rays within the voxels they cross through. Secondly, the definition of the rigid transformations applied to the $3 \mathrm{D}$ volumetric $\mathrm{CT}$ data with respect of the local reference of one of the projection planes allows the registration to be more accurate. Finally, two algorithms are used to solve the pose estimation problem: the hill climbing algorithm ${ }^{7}$ and a modified version, the dense hill climbing algorithm, which considers a combination of transformations. Besides, the proposed method allows to compensate, with confidence, a simulated rigid respiratory movement and thus could be used to obtain an augmented reality view to guide cardiologist navigate through complicated and small anatomical structures such as MAPCAS vessels. Although this study focused on the latter, the same procedure could be applied for any cardiovascular structures involving CT and Xray angiography. Our preliminary results are promising to automatically overlay a tomographic volumetric acquisition of such small structure as MAPCAs with X-ray angiography in a reasonable computation time to compensate a rigid respiratory motion during percutaneous interventions.

\section{ACKNOWLEDGEMENTS}

This study was funded by NSERC Discovery Grant program. The authors would like to thank the radiology and the cardiology department of Sainte-Justine Hospital for their continuous support and for providing clinical datasets. 


\section{REFERENCES}

[1] Griselli, M., McGuirk, S., Winlaw, D., Stumper, O., de Giovanni, J., Miller, P., Dhillon, R., Wright, J., Barron, D., and Brawn, W., "The influence of pulmonary artery morphology on the results of operations for major aortopulmonary collateral arteries and complex congenital heart defects," The Journal of thoracic and cardiovascular surgery 127, 251-258 (2004).

[2] Murthy, K., Krishnanaik, S., Coelho, R., Punnoose, A., Arumugam, S., and Cherian, K., "Median sternotomy single stage complete unifocalization for pulmonary atresia, major aorto-pulmonary collateral arteries and VSD-early experience.," European journal of cardio-thoracic surgery 16, 21-25 (1999).

[3] Markelj, P., Tomazevic, D., Likar, B., and Pernus, F., "A review of 3D-2D registration methods for imageguided interventions," Medical image analysis (2010).

[4] Westover, L., "Footprint evaluation for volume rendering," SIGGRAPH Computer graphics 24, 367-376 (1990).

[5] Siddon, R., "Fast calculation of the exact radiological path for a three dimensional CT array," Physics in medicine and biology 12(2), 252-255 (1985).

[6] Lemieux, L., Fish, D., Jagoe, R., Kitchen, N., and Thomas, D., "A patient to computed tomography image registration method based on digitally reconstructed radiographs," Medical physics 21, 1749-1760 (1994).

[7] Wein, W., Intensity based rigid 2D-3D registration algorithms for radiation therapy, Master's thesis, Technische Universität München (2003).

[8] Powell, M., "An efficient method for finding the minimum of a function of several variables without calculating derivatives," The computer journal 7(2), 155-162 (1964).

[9] Nelder, J. and Mead, R., "A simplex method for function minimization," The computer journal 7(4), 308$313(1965)$.

[10] Marquardt, D., "An algorithm for least-squares estimation of nonlinear parameters," SIAM Journal on applied mathematics 11, 431-441 (1963).

[11] Fletcher, R., "A new approach to variable metric algorithms," The computer journal 13, 317-322 (1970).

[12] Birkfellner, W., Seemann, R., Figl, M., Hummel, J., Ede, C., Homolka, P., Yang, X., Niederer, P., and Bergmann, H., "Wobbled splatting - a fast perspective volume rendering method for simulation of X-ray images from CT," Physics in medicine and biology 50, 73-84 (2005).

[13] Cosman, E., Rigid registration of MR and biplanar fluoroscopy, Master's thesis, Massachusetts Institute of technology (2000).

[14] Turgeon, G., Lehmann, G., Guiraudon, G., Drangova, M., Holdsworth, D., and Petersa T., "2D-3D registration of coronary angiograms for cardiac procedure planning and guidance," Medical physics 32, 3737-3749 (2005).

[15] Studholme, C., Hill, D., and Hawkes, D., "An overlap invariant entropy measure of 3D medical image alignment," Pattern recognition 32, 71-86 (1999).

[16] Jomier, J., Bullitt, E., Horn, M. V., Pathak, C., and Aylward, S., "3D-2D model to image registration applied to tips surgery," Medical image computing and computer-assisted interventation 9, 662-669 (2006).

[17] Liao, R., Duong, L., Sun, Y., and Kirchberg, K., "3D reconstruction of the coronary artery tree from multiple views of a rotational X-ray angiography," The international journal of cardiovascular imaging 26, 733-749 (2010).

[18] Rivest-Hénault, D., Duong, L., Cheriet, M., Lapierre, C., and Deschênes, S., "Semi-automatic segmentation of major aorto-pulmonary collateral arteries (MAPCAs) for image guided procedures," Proceeding of SPIE Medical imaging 7625, 76250B (2010).

[19] Lewis, J. and Jiang, S., "A theoretical model for respiratory motion artifacts in free-breathing CT scans," Physics in medicine and biology 54, 745-775 (2009). 